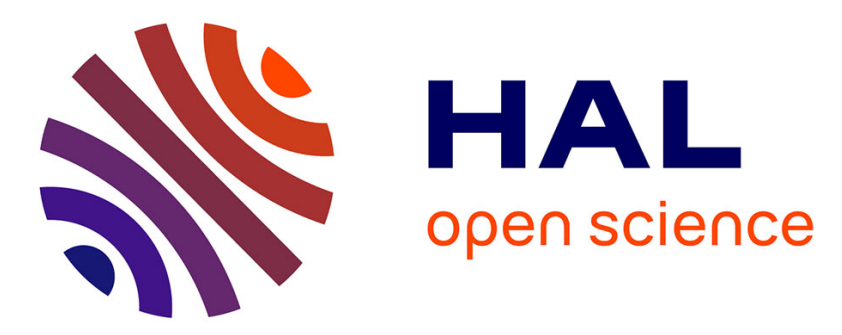

\title{
Transport of coarse particles in liquid foams: coupling of confinement and buoyancy effect
}

Florence Rouyer, Nicolas Louvet, Christelle Fritz, Olivier Pitois

\section{To cite this version:}

Florence Rouyer, Nicolas Louvet, Christelle Fritz, Olivier Pitois. Transport of coarse particles in liquid foams: coupling of confinement and buoyancy effect. Soft Matter, 2011, pp.4812-4820. 10.1039/c0sm01491e . hal-00698514

\section{HAL Id: hal-00698514 \\ https://hal-enpc.archives-ouvertes.fr/hal-00698514}

Submitted on 16 May 2012

HAL is a multi-disciplinary open access archive for the deposit and dissemination of scientific research documents, whether they are published or not. The documents may come from teaching and research institutions in France or abroad, or from public or private research centers.
L'archive ouverte pluridisciplinaire HAL, est destinée au dépôt et à la diffusion de documents scientifiques de niveau recherche, publiés ou non, émanant des établissements d'enseignement et de recherche français ou étrangers, des laboratoires publics ou privés. 


\title{
Transport of coarse particles in liquid foams: coupling of confinement and buoyancy effects
}

\author{
F. Rouyer, ${ }^{* a}$ N. Louvet, ${ }^{b}$ C. Fritz ${ }^{a}$ and O. Pitois ${ }^{a}$ \\ Received 14th December 2010, Accepted 16th March 2011 \\ DOI: 10.1039/c0sm01491e
}

\begin{abstract}
We investigate the behavior of coarse particles confined in foam channels during drainage. Results are reported for particle velocities measured at both microscopic (single foam channel) and macroscopic (foam) scales, as a function of the average velocity of the liquid flow and of the confinement parameter that is the ratio of particle diameter to the maximal particle diameter within channel cross-section. Thanks to numerical simulations, we show that velocities measured for small values of the confinement parameter cannot be understood with the commonly assumed theory for liquid flow in foam channels. Instead, better agreement is obtained by taking into account the characteristics of the flow in the films/ channel transitional areas. Finally, values for longitudinal dispersion coefficients are reported,

emphasizing effects of buoyancy on particles motions.
\end{abstract}

\section{Introduction}

Liquid foams are widely used in various basic applications as well as in elaborated industrial processes. In a large number of cases, particles are incorporated in the foam, and are either attached to liquid-gas interfaces or free to move between gas bubbles. Efficient attachment of hydrophobic particles allows for long-term stable foams to be produced. ${ }^{1}$ Incorporation of hydrophilic particles can also have suitable effects, such as the improvement of the mechanical properties. ${ }^{2}$ Note also that foams made with complex fluids, such as suspensions, intrinsically contain particles. In the latter case, optimization of a given process requires to predict the average particle velocity (relative to bubbles) and their dispersion within the foam. In froth flotation of mineral ores for example, the efficiency of the separation process is strongly affected by the amount of undesirable hydrophilic particles (unattached particles that compose the gangue) entrained with the liquid of the foam and ultimately recovered with attached valuable particles. The prediction of the behaviour of unattached particles within the foam is therefore of great interest. $^{3-6}$ This problem can be compared to particle transport through solid porous media, except that the pore surface is soft and generally 'mobile'. In this regard, a considerable amount of experimental and theoretical work on foam drainage has evidenced the effects of interfacial mobility and has shown that foam permeability varies over one order of magnitude depending on the surfactant used to stabilize the bubbles. ${ }^{7-10}$ In contrast,

${ }^{a}$ Université Paris-Est, Laboratoire de Physique des Matériaux Divisés et des Interfaces, CNRS FRE 3300, 5 bd Descartes, 77454 Marne la Vallée Cedex 2, France. E-mail: florence.rouyer@univ-mlv.fr; Fax: +3316095 72 97; Tel: +33160957263

${ }^{b}$ Laboratoire du Futur UMR 5258, 178 avenue du docteur Schweitzer, 33600 Pessac, France our knowledge on unattached particle motions in foams is restricted to a limited amount of experimental work.

Confocal microscopy was used by Koehler et al. ${ }^{11}$ to determine velocities of fluorescent particles in one Plateau border (microscopic scale). In that case, the particles were so small that their velocity was expected to be that of the liquid, allowing for velocity profiles to be measured. Note that this technique does not allow the measurement of velocity in channel's corners, whereas these areas are known to be subjected to specific flow conditions. ${ }^{12-14} \mathrm{At}$ the scale of the foam (macroscopic scale), Lee et al. ${ }^{15}$ have shown that the dispersion coefficient characterizing the transport of such small particles is very close to that of the liquid.

For larger particles transported in a two-dimensional rising foam, Bennani et al. ${ }^{16}$ have reported significant confinement effects. It has been shown that a particle can be strongly slowed down with respect to the liquid due to their interactions with bubble surfaces. For these particles, the gravity force can become significant and particulate motion therefore results from viscous drag due to interstitial liquid flow combined with the sedimentation motion. In this regard, the sedimentation motion of particles confined in foam channels has been investigated in detail, ${ }^{17,18}$ providing expressions for the particle velocity as a function of the particle/channel size ratio, the surface shear viscosity characterizing bubble surfaces and the particle position within the channel's cross-section.

Note also that particles with a diameter larger than the size of constrictions in the foam channel network can be permanently trapped, so that the particle velocity (relative to the bubbles) vanishes. The capture criterion proposed recently by Louvet et al. ${ }^{19}$ enables to predict the critical size below which a particle is free to move in interstices between foam bubbles.

Other works have focussed on drainage of foams made with colloidal suspensions. ${ }^{21-23}$ It has been emphasized that drainage 
laws do not predict the measured drainage velocity. The authors often refer to confinement and collective effects, expressed in terms of trapping of particle aggregates in foam channels. Note that this effect could also be expressed in terms of appearance of a yield stress for the interstitial suspension. In this regard, the interstitial flow of suspensions in foams has been shown to be prevented thanks to the yield stress properties. ${ }^{24,25}$ Obviously, the prediction of particle aggregation in the channel network requires better insight into the motions of individual particles. This understanding is still lacking, even for the simplest situation of a single nonBrownian sphere convected in the foam channel network.

In the present work, we investigate the behavior of coarse particles confined in foam channels during drainage. We report results obtained for particle velocities at both microscopic (single foam channel) and macroscopic (foam) scales. In these experiments, particular attention is paid to the control of the ratio of particle size to the radius of passage of the channels, providing thereby a significant insight into the effect of particle confinement on this problem. These experiments coupled with numerical simulations question the theory for liquid flow in foam channels and indicate that the characteristics of the flow in the films/channel transitional area have to be taken into account. The results also emphasize the marked effect of buoyancy on the dispersion process through the foam.

\section{Modeling}

\subsection{Liquid flow in a foam channel}

Foam channels, the so-called Plateau borders, are formed by the merging of foam films when they intersect symmetrically three by three. The channel's cross-section is then bounded by three tangentially connected circular arcs of radius $R_{\mathrm{PB}}$ and angle $\pi / 3$, as depicted in Fig. 1. Channel's interfaces connect to a thin foam film within a transitional area located at each corner of the channel's cross-section.

In a pioneer work, Leonard and Lemlich ${ }^{12}$ have proposed a simple model for the liquid flow in such channels. The liquid flow through an infinite Plateau border, with axis along $z$-axis in the Cartesian space $(x, y, z)$, is assumed to be uniaxial and stationary, characterized by a velocity profile $v(x, y)$ along the $z$ axis. Typical Reynolds number for the liquid is assumed to be small and the Stokes equation is used:

$$
\Delta v=\frac{1}{\mu} \frac{\mathrm{d} p}{\mathrm{~d} z}
$$

where $\mu$ is the liquid viscosity and $p$ is the liquid pressure (in case of uniform foam channel, the pressure gradient is equal to gravity forces). Partial mobility of channel's interfaces is obtained by balancing the bulk viscous stress with the surface viscous stress on the circular boundary:

$$
\text { n. } \nabla v=\frac{\mu_{\mathrm{s}}}{\mu} \Delta_{\mathrm{s}} v
$$

where $\mu_{\mathrm{s}}$ is the shear surface viscosity and $\Delta_{\mathrm{s}}$ is the surface Laplacian and $\boldsymbol{n}$ is the unit vector normal to the surface pointing out of the channel. The Boussinesq number compares the surface viscous stress to the bulk viscous stress: $B o=\mu_{\mathrm{s}} / \mu R_{\mathrm{PB}} \cdot{ }^{12}$ Finally, it is assumed that the liquid velocity vanishes at each corner of the channel's cross-section (to distinguish better from the $V_{\mathrm{C}} \neq$ 0 case considered subsequently).

Lemlich's model ignores the contribution of surface elasticity in the behavior of channel's interfaces as liquid flows. Very recently, the Marangoni effect has been introduced in the modeling of the flow. ${ }^{14}$ The simple picture of the model is the following: surfactant is convected on the central part of the circular interfaces due to the viscous stress from the bulk, inducing surface tension gradients between channel's ends (inletoutlet) so that Marangoni flows take place in the transitional area at proximity to the channel corners that join channels to films. This mechanism can be expressed in terms of a counterflow velocity $V_{\mathrm{C}}$ in the transitional area, which has been shown to be proportional to the average velocity of the liquid flowing in the bulk of the channel, with a typical coefficient of a few units. ${ }^{14}$

\subsection{Particle transport in a foam channel}

The motion of a buoyant particle in a foam channel results from the combination of the sedimentation motion and the convection motion due to liquid flow. In opposition to the case of particles

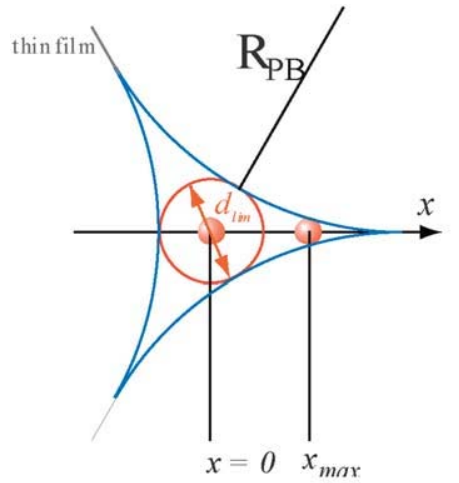

(a)

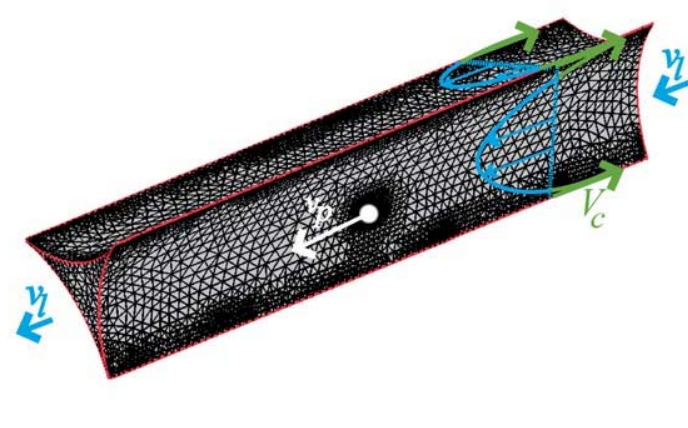

(b)

Fig. 1 (a) Sketch of the cross-section of a foam channel with the geometrical limit diameter $\left(d_{\text {lim }}\right)$ for a transported particle. The position of a small particle is measured along the $x$-axis: $x=0$ at the center of the channel and $x=x_{\max }$ at the corner. (b) Example of mesh used to compute the velocity of a sphere $\left(v_{\mathrm{p}}\right)$ in a Plateau border with an average liquid velocity $v_{1}$. The velocity profile for the fluid interface is also presented as an illustration: $V_{\mathrm{c}}$ is the liquid velocity at the corner of the channel and one of the parameters of the simulations. 
close to solid wall for which rolling motion is observed, no rolling motion is observed in the present case of mobile interface. Indeed, rolling motion is induced by large shear stresses on the side facing the wall in opposition to a low shear stress on the opposite side. In the presence of mobile interface, the difference in shear stresses on different parts of the particle is considerably diminished. For this reason, we neglect rotation of the particle.

For vanishing liquid flow rates in the channel, the particle motion is the pure sedimentation motion already studied in detail. ${ }^{17,18}$ For a vertical Plateau border, the sedimentation velocity can be expressed as a function of the confinement parameter $\lambda$, which compares the particle diameter $d$ with the diameter of the circle inscribed in the channel's cross-section, $d_{\text {lim }}$ (see Fig. 1a), the Boussinesq number and the position of the particle within the channel's cross-section. For foam (inclined) Plateau borders, it has been numerically shown that particles settle along the lowest corner in the Plateau border's cross-section, so that the sedimentation velocity can be expressed with $\lambda$ and $B o$ only:

$$
V_{\text {sed }}=h(\lambda) g\left(B o^{\prime}\right) V_{\text {St }} \sin (\theta)
$$

where $\theta$ is the angle of inclination of the Plateau border with respect to the horizontal plane, $h(\lambda)=\left(1-1.488 \lambda+0.535 \lambda^{2}\right), g\left(B o^{\prime}\right)=(4.3$ $\left.+B o^{\prime}\right) /\left(1.8+2.2 B o^{\prime}\right)$ and $V_{\mathrm{St}}=\Delta \rho g d^{2} / 18 \mu$, with the particulate Boussinesq number, $B o^{\prime}=\mu_{\mathrm{S}} / \mu d=B o / 2 \lambda(2 / \sqrt{3}-1)$ and the confinement parameter $\lambda=d / d_{\text {lim }}=d / 2 R_{\mathrm{PB}}(2 / \sqrt{3}-1)$, within the ranges $0.002-20$ and $0.1-0.8$ for $B o^{\prime}$ and $\lambda$ respectively.

For negligible buoyancy effects, the particle velocity can be determined from numerical simulations for the particle suspended in the liquid flowing through the Plateau border. We use COMSOL Multiphysics software to solve the fluid flow through a Plateau border channel of radius $R_{\mathrm{PB}}$ and length $4 R_{\mathrm{PB}}$. A sphere is set at midway in the channel $\left(2 R_{\mathrm{PB}}\right)$ and its position within the channel's cross-section is referred to the coordinate $x$ along one axis of symmetry of the cross-section (see Fig. 1). We use an "auto" extra-fine mesh refined near the particle (maximum element size $=0.02$ and growth rate $=1.1$ ) and the corner of the $\mathrm{PB}$ (maximum element size $=0.05$ and growth rate $=1.05$ ) as illustrated in Fig. 1b. We consider dimensionless Stokes equations (fluid density and viscosity are respectively set to zero and one in "the Fluid Dynamic, Incompressible Navier-Stokes" user interface and $R_{\mathrm{PB}}=1$ ). The simulations are run in the frame of the particle and zero velocity is imposed at the surface of the particle (no-slip conditions). Fluid velocities at the entrance and at the outlet of the channel are constrained to the constant parameter $-V_{\mathrm{p}}+V_{1}$. Fluid velocities at the edges of the channel are constrained to the parameter $-V_{\mathrm{p}}-V_{\mathrm{c}}$ where $V_{\mathrm{c}}$ is the counterflow velocity imposed at the three corners of the channel. The fluidity of the interface is modeled using weak terms for balancing the bulk viscous stress with the surface viscous stress on the circular boundary. Moreover $V_{1}$ is chosen such that the effective average liquid velocity, is set to one ( $\frac{1}{A_{\mathrm{PB}}} \int_{A_{\mathrm{PB}}} v H(v) \mathrm{d} A=1$, where $H$ is the Heaviside step function) for every value of $B o$ and $V_{\mathrm{c}}$, Thus, the numerical simulations are non-dimensionalised on the scale $v_{1}$. Note that the counterflow does not contribute to the mean flow rate through the foam because it is balanced by an inverse flow in the film (that induces thickening of the films) and that counterflow (where $v<0$ below the red line $c f$. Fig. 2a) is limited to a region in the corner that increases with $B o$. The dimensionless force $F_{\mathrm{V}}$ exerted on the sphere by the viscous flow is calculated from the integral of the Lagrange multiplier along the axis of the channel. For given $x, \lambda$, $V_{\mathrm{c}}$ and $B o$, a parametric simulation that varied $V_{\mathrm{p}}$ is run to determine the value $V_{\mathrm{p}}=\xi\left(x, \lambda, B o, V_{\mathrm{c}}\right)$ for which $F_{\mathrm{V}}=0$. The relation between the velocity of a non-buoyant particle and the average liquid velocity is then deduced: $v_{\mathrm{p}}=v_{1} \xi\left(x, \lambda, B o, V_{\mathrm{c}}\right)$ where $\xi$ is a dimensionless function. An illustration of calculations made to determine $\xi$ is presented in Fig. 2a for parameter values of $V_{\mathrm{c}}$ and $B o$ that are consistent with the values reported in the literature and later in this paper. As it can be observed in Fig. 2a, the particle velocity is approximately equal to the velocity of the undisturbed fluid at its centre. For creeping flow, Faxen's law predicts the velocity of a non-buoyant spherical particle from the velocity of the fluid in the channel (undisturbed by the presence of the particle): $v_{\mathrm{p}}(x)=v(x)+\frac{a^{2}}{6} \Delta v$. The present simulations agree very well with Faxen's law ( $\pm 0.002 v_{1}$ absolute error) for the centred particle. However, for the particle at a corner, Faxen's law overestimates our numerical results and all the more that the particle is small (up to $0.07 v_{1}$ absolute error for the smallest particle). Smaller the particle size, smaller is the distance to the corner where we impose the velocity, thus, we attribute the discrepancy to particle-corner hydrodynamic interactions that are not considered in the Faxen theory similarly to particle-wall hydrodynamic interactions evidenced in the Poiseuille flow. ${ }^{20}$

For buoyant particle, the settling velocity of the particle $V_{\text {sed }}$ superimposes to convection due to liquid entrainment. Due to the linearity of Stokes equation, the velocity of a non-rotating buoyant particle in a creeping flow is expected to be equal to the sum of its settling velocity and of the velocity of the equivalent non-buoyant particle convected by the flow. Few simulations with dimensional parameters, that correspond to the physically realistic parameter values (density and viscosity fluid, surface shear viscosity, size of Plateau border), have been run to determine the particle velocity in a stationary state for which gravitational force is balanced by the flow, we thus verify the additivity of sedimentation and convective velocities for various particle densities and liquid velocities for the range of parameters considered in this work. Fig. $2 b$ shows how the gravity force affects the particle motion at low liquid velocity. The particle velocity is thus given by the following expression:

$$
v_{\mathrm{p}}=V_{\text {sed }}+v_{1} \xi\left(\lambda, B o, V_{\mathrm{c}}\right)
$$

\subsection{Liquid and particle transport in the foam}

In the foam, Plateau borders are connected four by four and form the liquid network in which liquid and particles are transported. Although this network can expand and retract depending on the interstitial liquid flow rate through the foam, one can relate the microscopic parameters, i.e. the average liquid velocity and the confinement parameter, to the liquid volume fraction and bubble size.

Let us consider a liquid foam of uniform liquid fraction $\varepsilon$ during gravity drainage. The interstitial liquid velocity in the macroscopic direction of transport can be written as: ${ }^{26}$ 

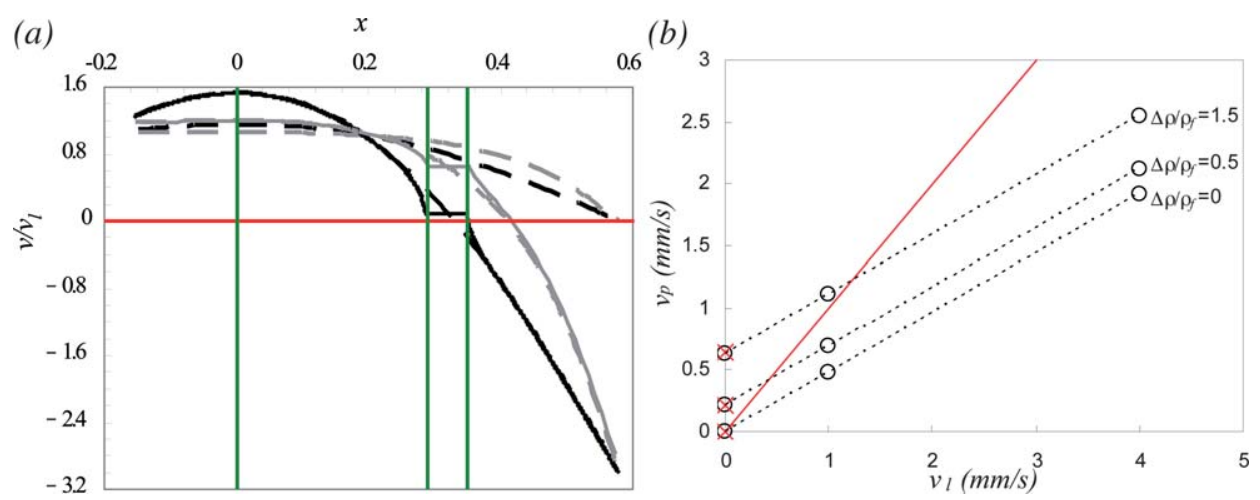

Fig. 2 Numerical simulation for a particle transported through a vertical Plateau border channel, along the closest axis from a channel corner. The confinement parameter $\lambda=0.2$. (a) Effect of counterflow velocity: liquid velocity profiles ( $x$-axis refers to Fig. 1a) for $B o=0.01$ (grey) and $B o=0.1$ (black) without (dotted lines) or with (continuous lines) a non-buoyant particle, with $V_{\mathrm{c}}=0$ and $V_{\mathrm{c}}=3 v_{1}$. The vertical lines illustrate the center of the cross-section $(x=0)$ and the area occupied by the particle (flat velocity profile in this section). (b) Effect of particle buoyancy: particle velocity versus liquid velocity with $R_{\mathrm{PB}}=400 \mu \mathrm{m}, \mu=0.001 \mathrm{~Pa} \mathrm{~s}, \mu_{\mathrm{S}}=10^{-8} \mathrm{~kg} \mathrm{~s}^{-1}, \rho_{\mathrm{f}}=1000 \mathrm{~kg} \mathrm{~m}^{-3}$ and $V_{\mathrm{c}}=3 v_{1}$, for several values of the reduced particle density. The results are compared to eqn (3) $\left(v_{1}=0\right.$, red times symbol). Dotted lines correspond to a linear fit obtained with a slope $=0.477 \pm 0.1 \%$ and a coefficient of regression $R=1$. Continuous line corresponds to the liquid velocity—slope $=1$.

$$
v_{1}=\frac{\tilde{K}(\varepsilon)}{\varepsilon} \frac{\rho g R_{\mathrm{b}}^{2}}{\mu}
$$

where $R_{\mathrm{b}}$ is the bubble radius and the dimensionless foam permeability $\tilde{K}(\varepsilon)=K(\varepsilon) / R_{\mathrm{b}}^{2}$ depends on the foaming solution i.e. depends on the mobility of the surface and so on the $B$ o number. ${ }^{27}$ For example, for TTAB solution (used in the experiments): $\tilde{K}(\varepsilon)=4 \varepsilon^{3 / 2} /\left[1700\left(1-2.7 \varepsilon+2.2 \varepsilon^{2}\right)^{2}\right] .{ }^{28}$

The confinement parameter expresses as a function of the diameter of passage $d_{\text {lim }}$ of constrictions in the channel network. According to the expression for $d_{\text {lim }}$ published recently, ${ }^{19} \lambda$ can be written as:

$$
\lambda\left(R_{\mathrm{b}}, \varepsilon\right)=\left(\frac{d}{2 R_{\mathrm{b}}}\right)\left(\frac{2 R_{\mathrm{b}}}{d_{\mathrm{lim}}}\right)=\left(\frac{d}{2 R_{\mathrm{b}}}\right) \frac{1+0.57 \varepsilon^{0.27}}{0.27 \sqrt{\varepsilon}+3.17 \varepsilon^{2.75}}
$$

Note that for low liquid fractions, i.e. $\varepsilon \leq 0.02$, a simpler expression can be used to relate liquid fraction and the diameter of passage that assumed Kelvin cell geometry for a bubble, ${ }^{27}$ so that $\lambda$ becomes:

$$
\lambda\left(R_{\mathrm{b}}, \varepsilon\right)=\frac{d \varepsilon^{-1 / 2}}{2 \sqrt{3}(2 / \sqrt{3}-1) R_{\mathrm{b}}}
$$

Another parameter of interest for particle transport is the coefficient of dispersion $D_{\mathrm{L}}$ of the particles in the longitudinal direction (direction of macroscopic transport). This coefficient accounts for particle velocity fluctuations in the longitudinal direction with respect to the average particle velocity. For particles smaller than $5 \mu \mathrm{m}$, it has been shown by Lee et al. that particle dispersion is similar to that of the liquid. ${ }^{15}$ As presented in the Introduction, we recall that there are no experimental data for dispersion coefficients of larger particles.

\section{Experimental}

\subsection{Materials}

An aqueous foaming solution is prepared with TTAB concentrated at $3 \mathrm{~g} \mathrm{~L}^{-1}$ i.e. three times greater than the critical micelle concentration that corresponds to low surface shear viscosities: $2-4 \times 10^{-5} \mathrm{~g} \mathrm{~s}^{-1} .^{11,9}$ The bulk shear viscosity $\mu$ and density $\rho$ of solutions are equal to those of pure water. Surface tension $\sigma=37$ $\mathrm{mN} \mathrm{m}^{-1}$.

Two types of solid particles are used: polystyrene beads later referred to as non-buoyant particles (Duke Scientific Corporation, density $\rho_{\mathrm{S}}=1050 \mathrm{~kg} \mathrm{~m}^{-3}$, diameter $d=40 \mu \mathrm{m}, 80 \mu \mathrm{m}$ and $140 \mu \mathrm{m})$ and silica beads later referred to as buoyant particles (Duke Scientific Corporation, density $\rho_{\mathrm{S}}=2450-2500 \mathrm{~kg} \mathrm{~m}^{-3}$ ). Diameters, $d$, are in the range $15-140 \mu \mathrm{m}$ and hand checked such that size dispersion is small: $\Delta d / d<5 \%$.

\subsection{Single vertical channel experiment}

The setup has been described in detail in ref. 17 . We briefly recall that the vertical channel is obtained by withdrawing a dedicated frame from a reservoir containing the foaming solution. The frame consists of a vertical metallic tube on which three vertical rods (diameter $1 \mathrm{~mm}$ ) are fixed at the bottom (like a tripod). Due to capillary forces, a Plateau border is formed at the merging line of the three verticals soap films supported by the frame and stabilized with surfactants contained in the solution. The frame is accurately positioned with reference to the reservoir to easily adjust the length of the channel (typical lengths are in the range 5-10 $\mathrm{mm}$ ).

The inner diameter of the tube is $10 \mathrm{~mm}$ and a circular outlet of diameter $1 \mathrm{~mm}$ is opened at its lower part: it is used to deliver the particles one by one through the channel and the liquid at a constant flow rate $q$ varied within the range $1-100 \mathrm{~mm}^{3} \mathrm{~min}^{-1}$. A dedicated delivery system was developed to introduce the particles into the foam channels without additional liquid flow. The particles are first mixed with the foaming solution and placed in a modified 3-path valve, ensuring their transfer to the feed tube where they are allowed to settle. Images of the channel were grabbed during the measurements through windows in the cell cover at the rate of 30 frames $\mathrm{s}^{-1}$. The successive positions occupied by a sphere along the channel axis are then determined by image processing procedures. Another window was also placed at the bottom of the liquid reservoir. By illuminating the 
channel from the top (through the tube) and using a high speed camera equipped with an appropriate lens, pictures of the channel cross-section could be obtained during particle motion. The pictures are then used to correlate the particle position within the channel's cross-section with the velocity of the corresponding particle, and to estimate the radius of curvature of the Plateau border and the cross-sectional area $A_{\mathrm{PB}}$. The average liquid velocity is therefore obtained with the relation: $v_{1}=q / A_{\mathrm{PB}}$. Measured liquid velocities are within the range $0.5-50 \mathrm{~mm} \mathrm{~s}^{-1}$, and channel width (equal to $R_{\mathrm{PB}}$ ) is close to $450 \mu \mathrm{m}$. With the values obtained for $R_{\mathrm{PB}}$ and $v_{1}$, the typical values for the Boussinesq number and the Reynolds number are respectively $B o \approx 0.07 \pm 0.05$ and $0.1 \leq R e \leq 3.5$ where $\operatorname{Re}=\frac{\rho_{\mathrm{f}} v_{1} d_{\text {lim }}}{2 \mu}$. Note also that for silica particles, the maximal particulate Reynolds number $\left(R e_{\mathrm{p}}\right)$ is equal to 3.4 for the largest particle and less than or equal to 0.1 for particles smaller than $45 \mu \mathrm{m}$ in diameter.

\subsection{Foam experiment}

Steady drainage experiments are conducted inside vertical Plexiglas cylindrical cells whose height is larger than $500 \mathrm{~mm}$ and section $S$ is equal to 29 or $9 \mathrm{~cm}^{2}$, depending on the bubble size, in such a way that the cell diameter is at least equal to 20 times the bubble diameter (Fig. 3). The cell is first filled with the foaming solution and bubbles are generated by blowing a mixture of nitrogen and $\mathrm{C}_{6} \mathrm{~F}_{14}$ through a needle in the liquid. $\mathrm{C}_{6} \mathrm{~F}_{14}$ gas is used with the aim to prevent foam coarsening. For each experiment, the bubbles are monodisperse and their diameter $2 R_{\mathrm{b}}$ is equal to $3 \mathrm{~mm}( \pm 6 \%)$. A lid is used to ensure water vapour saturation inside the foam cell. After a long time of drainage $(\sim 20 \mathrm{~min})$ a very dry foam $(\varepsilon \approx 0)$ is obtained, and a constant liquid flow rate $Q$ is then imposed at the top; $Q$ is varied within the range 5-70 $\mathrm{ml} \mathrm{min}^{-1}$. A CCD camera records the evolution of position of the front between the upper wet foam and the dry foam. The front velocity $v_{1}$ is known to be constant and related to the liquid fraction of the upper wet foam by: $\varepsilon=(Q / S) / v_{1}$. Measured liquid fractions remain smaller than 0.04 in any case.

The particles are injected one by one, at a height $H$ approximately equal to $10 \mathrm{~cm}$ from the liquid bath (Fig. 3). We measure the time $t$ over which the particles flow toward the bottom of the foam. The particle velocity is then calculated as: $v_{\mathrm{p}}=H / t$.

We determine the coefficient of dispersion $D_{\mathrm{L}}$ of the particles in the longitudinal direction (same direction as the transport) as:

$$
D_{\mathrm{L}}=\frac{\left\langle v_{\mathrm{p}}\right\rangle^{2}}{2} \frac{\left\langle\Delta t^{2}\right\rangle}{\langle t\rangle}=\frac{H^{2}}{2} \frac{\left\langle\Delta t^{2}\right\rangle}{\langle t\rangle^{3}}
$$

where \langle\rangle denotes average over measurements for which the liquid velocity is fixed and $t$ is the total transport time for one particle through the foam, so $\left\langle\Delta t^{2}\right\rangle$ is the variance of $t$.

\section{Results and discussion}

First, we present in Fig. 4 typical results obtained for both polystyrene and silica particle velocities as a function of the average liquid velocity through the vertical Plateau border channel. Presented results correspond to two values of the confinement parameter: $\lambda=0.2$ and $\lambda=0.85$ (the particles are respectively $40 \mu \mathrm{m}$ and $140 \mu \mathrm{m}$ in diameter). In any case, the

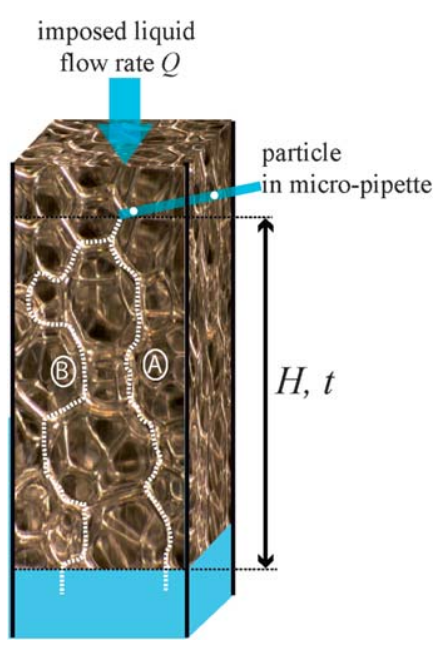

Fig. 3 Sketch of the foam cell that illustrates the foam drainage experiment, the injection of a single particle and two different paths A and B through the foam.

average particle velocity increases with the liquid velocity. For the highest value of $\lambda$, both types of particles have velocity values close to the liquid velocity, except for the silica particles at very low liquid velocity. Instead, for $\lambda=0.2$, particle velocity values exhibit drastic differences depending on the particle type: silica particle velocities are only weakly scattered around the average value, whereas polystyrene particle velocity values are extremely scattered. For this latter case, $v_{\mathrm{p}}$ can be either as high as the liquid velocity, or close to zero. For both particle types, however, the average particle velocity increases almost proportionally to the liquid velocity.

The effect of the confinement parameter is presented in Fig. 5. In order to compare this effect for both types of particles, we remove the contribution of sedimentation, which is the major contribution for large silica particles at low liquid velocity. Thus, we report in Fig. $5\left(v_{\mathrm{p}}-V_{\text {sed }}\right)$ normalized by the liquid velocity as a function of $\lambda$ for both types of particles. Fig. 5 supplements the first insight provided by Fig. 4: data for polystyrene beads are highly scattered for small $\lambda$ values and the scatter reduces as $\lambda$ increases. This effect does not affect results for silica particles, for which scatter remains limited whatever the value of $\lambda$. Another interesting feature can be gathered for the particulate motions: when the contribution of sedimentation is removed for buoyant particles, the resulting velocity is always smaller than the average liquid velocity; in contrast, results for non-buoyant particles exhibit particle velocities that can be significantly larger than the liquid velocity. This effect is all the more likely that $\lambda$ value is high. Finally, the lowest velocity value for polystyrene particles is close to the average value of velocities measured for silica particles for the same $\lambda$ value.

Visual inspections at the bottom of the vertical Plateau border during measurements indicate that fastest particles occupy the central position in the cross-sectional area, whereas slowest particles always move along channel's corners, as presented in images inserted in Fig. 5. The visualization also revealed that silica particles are always located at a corner of the channel. Obviously, this specific location of silica particles when moving through the Plateau border channel can be attributed to the 

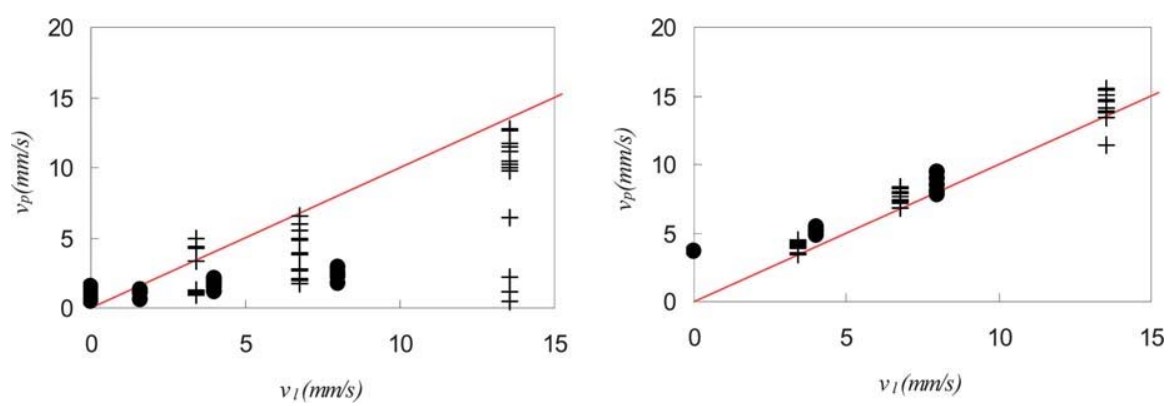

Fig. 4 Typical particle velocity of polystyrene (plus symbols) and silica beads (round symbols) as a function of the average liquid velocity through a vertical Plateau border for two values of the confinement parameter, $\lambda=0.2$ (left) and $\lambda=0.85$ (right). The line corresponds to $v_{\mathrm{p}}=v_{1}$.

effect of the gravity force: buoyant particles have time to settle (vertically) and to migrate towards inclined walls of the injection device before they enter the Plateau border channel. This effect can be also expected for motions of buoyant particles in foams and it will be discussed later.

Now, we compare results presented in Fig. 5 with theoretical predictions obtained through numerical simulations. Both minimal and maximal values for the theoretical velocity are plotted in Fig. 6a for non-buoyant spheres, for two values of the Boussinesq number (0.01 and 0.1) enclosing the expected experimental value, and two values for counterflow velocity: $V_{\mathrm{c}}=0$ (Lemlich's assumption) and $V_{\mathrm{c}} / v_{1}=3$. Numerical results clearly show that theoretical values with $V_{\mathrm{c}}=0$ are not compatible with experimental data. Indeed, for $\lambda=0.2$, the predicted value for the minimum particle velocity is by far larger than the corresponding measured values for both polystyrene and silica beads. Predicted maximum velocity is lower than the measured one, whatever the value used for $B o$ in the simulation. As a result, the theoretical velocity deviation, i.e. difference between maximum and minimum, (0.4) is almost four times smaller than the measured deviation for polystyrene beads (1.5). On the other hand, the introduction of counterflow velocity at each corner of the channel cross-section, i.e. $V_{\mathrm{c}} \neq 0$, allows for a better agreement to be obtained for $\lambda=0.2$. Calculated

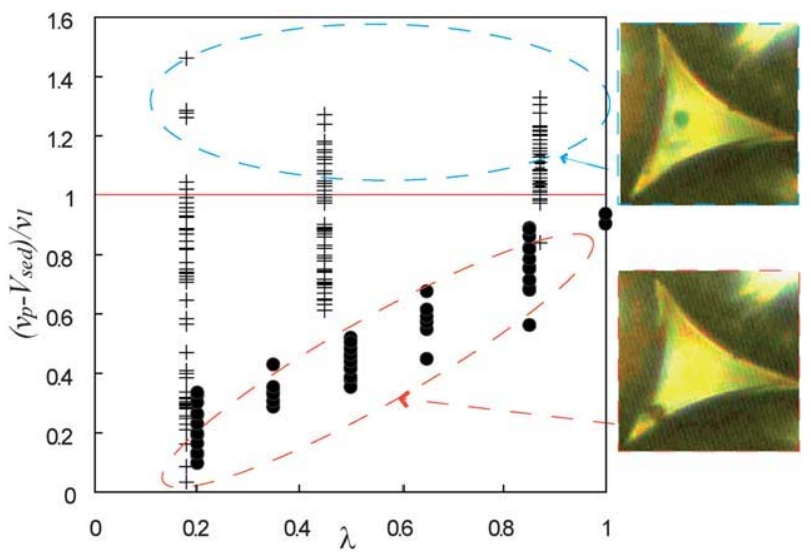

Fig. 5 Convective contribution in measured particle velocity for particles transported through a single vertical Plateau border: polystyrene beads (plus symbols) and silica beads (rounded symbols) as a function of the confinement parameter $\lambda$. Dash ovals connected to images underline the data obtained for two typical positions in the cross-section: close to the center (top) and close to the corner (bottom). minimum particle velocities for several values for $B o$ and $V_{\mathrm{c}}$ are reported in Fig. 6b with the measured minimum velocity. We found that a counterflow velocity equal to $V_{\mathrm{c}}=3 v_{1}$ allows for experimental values to be properly described. This value falls directly within the range of expected values. ${ }^{14}$ Note that the counterflow velocity affects the minimum particle velocity because the slowest particles move along channel's corners-thus close to the counterflow area-but it also affects the maximum particle velocity because the counterflow modifies significantly the liquid velocity throughout the channel's cross-section, including the central position corresponding to the fastest particles. This effect is all the more efficient that the counterflow reduces the hydrodynamic channel's cross-section, and all the more that $V_{\mathrm{c}}$ is large. This effect contributes to increase the velocity of particles located in the central part of the cross-section (as illustrated in Fig. 2) and thus contributes to increase the deviation between the fastest and the slowest particles. In this regard, the agreement between measured velocities and calculated ones, for $\lambda=0.2$, is satisfactory.

However, the velocity of larger particles is not properly described, whatever the value for $V_{c}$, emphasizing the limits of the modeling for particle transport. Obviously, the presence of a large particle within the channel's cross-section modifies deeply the balance of bulk flow and counterflow in the transitional area. By imposing a constant coefficient for the counterflow velocity at the channel's corner, the drag effect induced by counterflow is underestimated as the size of the transported particle increases. We think that a more complete numerical simulation, including surfactant transport and Marangoni stresses in the transitional area, is required to understand all particle velocities reported in Fig. 5. This disagreement is also evident in Fig. 4b, where eqn (4) overestimates significantly the velocity of large particles: this equation would be presented by a line intercepting the $y$-axis at $V_{\text {sed }}>0$ with a slope $\xi$, that depends on the parameter values of $B o$ and $V_{\mathrm{c}}$ but is always larger than one (cf. Fig. 6a).

Now, we present the results for the transport of particles in the foam. Fig. 7 shows typical particle velocities as a function of liquid velocity measured for silica beads with diameters equal to $22.5 \mu \mathrm{m}, 42.5 \mu \mathrm{m}$ and $85 \mu \mathrm{m}$. For every particle size, the particle velocity increases with the liquid velocity. For small particles, we observe that most of the data spread below the line $v_{\mathrm{p}}=v_{1}$ and that reported velocity can be less than one-third of the average liquid velocity. The average value for the particle velocity is smaller than the average liquid velocity. This effect is in agreement with results obtained at a microscopic scale. In contrast, 

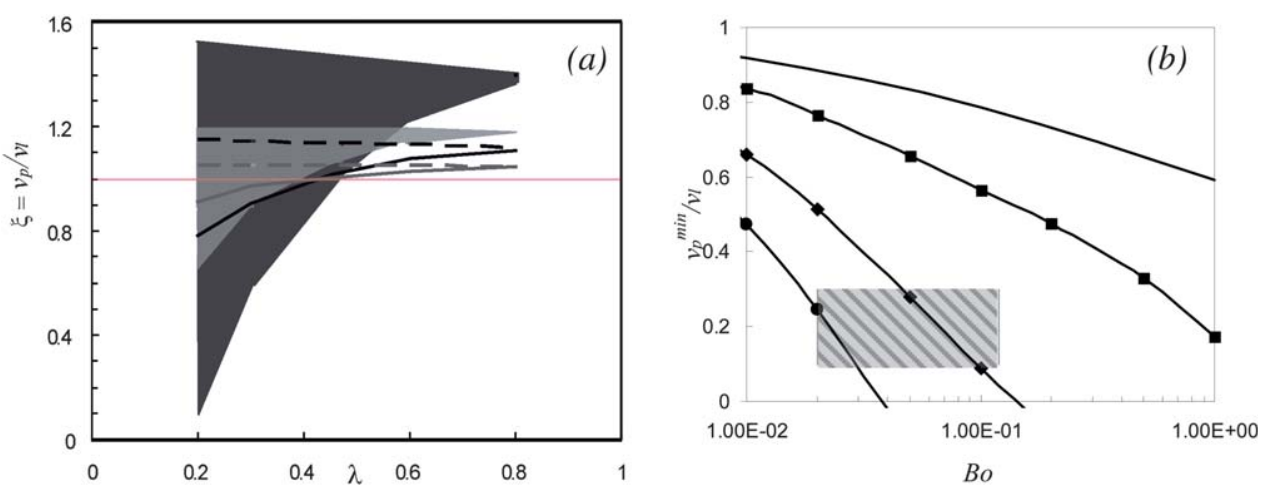

Fig. 6 (a) Numerical values for the normalized particle velocity as a function of confinement parameter $\lambda$ : minimal (continuous lines) and maximal (dashed lines) particle velocities for $B o=0.1$ (black lines) and 0.01 (grey lines) assuming no counterflow velocity, and range of particle velocities for $B o=0.1$ (dark grey area) and 0.01 (light grey area) assuming a counterflow velocity equals to 3 times the average liquid velocity. (b) Numerical values for the normalized particle velocity of particles moving along a channel's corner as a function of the Boussinesq number, for $\lambda=0.2$ and for several values for the normalized counterflow velocity $V_{\mathrm{c}}^{*}: V_{\mathrm{c}}^{*}=0$ (no symbols), $V_{\mathrm{c}}^{*}=1$ (square symbols), $V_{\mathrm{c}}^{*}=3$ (diamond symbols), $V_{\mathrm{c}}^{*}=5$ (circle symbols). Dashed area corresponds to the range of experimental data.

velocities of the largest particles are larger than the average liquid velocity. This behavior can be compared to results obtained at a microscopic scale, removing the contribution of sedimentation. The resulting velocity $\left(v_{\mathrm{p}}-V_{\text {sed }}\right) / v_{1}$ is plotted as a function of the confinement parameter in Fig. 8. This plot shows that the contribution of sedimentation is not responsible for the higher values measured for the largest particles. Qualitatively, this result is in agreement with those obtained at the scale of the single vertical Plateau border (Fig. 5), but quantitatively, velocities measured at foam scale are larger than those measured in the single channel. Therefore, one can deduce that this deviation results from a network effect. Basically, paths of large particles are not equivalent to paths of small particles and liquid, due to the buoyancy force, and independently of the geometrical effect of the confinement parameter. In other words, large particles have time to settle when passing through junctions between two neighbouring channels, resulting in more vertical paths through the channel network. Obviously, a relevant parameter for this effect can be expressed in comparing the particle convection time through a channel junction of typical length $\alpha R_{\mathrm{PB}}$ (with $\alpha$ a constant characteristic of a junction size of the order of unity), with the time corresponding to the vertical settling of the particle over a length $\ell$ allowing particle swapping between streamlines entering different channels. $\ell$ can be defined as $\ell=\alpha^{\prime} d_{\lim } / 2=\alpha^{\prime}(2 / \sqrt{3}-1) R_{\mathrm{PB}}$ (with $\alpha^{\prime}$ of the order of unity). We note that $\alpha$ and $\alpha^{\prime}$ might depend on the liquid volume fraction and on $\lambda$ (large particle have access to the smaller cross-section area than small particle) but at first order we estimate $\alpha / \alpha^{\prime} \approx 1$. The resulting time ratio is thus $(2 / \sqrt{3}-1) v_{1} / V_{\mathrm{St}} \approx 0.16 v_{1} / V_{\mathrm{St}}$. Particle velocities are now plotted as a function of $0.16 v_{1} / V_{\mathrm{St}}$ in Fig. 8b. Data obtained for all particle sizes sort in a coherent way as a function of this parameter: $v_{\mathrm{p}}-V_{\text {sed }}>v_{1}$ when $0.16 v_{1} / V_{\mathrm{St}}<1, v_{\mathrm{p}}-V_{\text {sed }} \approx v_{1}$ when $0.16 v_{1} / V_{\mathrm{St}} \approx 1$ and $v_{\mathrm{p}}-V_{\text {sed }}<v_{1}$ when $0.16 v_{1} / V_{\mathrm{St}}>1$. Note also that this parameter is an approximate criteria to distinguish particle that can $(<1)$ or cannot $(>1)$ settle inside channel junctions, and that it can be expressed as $0.16 v_{1} / V_{\mathrm{St}} \approx\left(9.7 / \lambda^{2}\right)\left(\tilde{K} / \varepsilon^{2}\right)$ $(\rho / \Delta \rho)$ emphasizing the combined effects of both confinement and buoyancy.

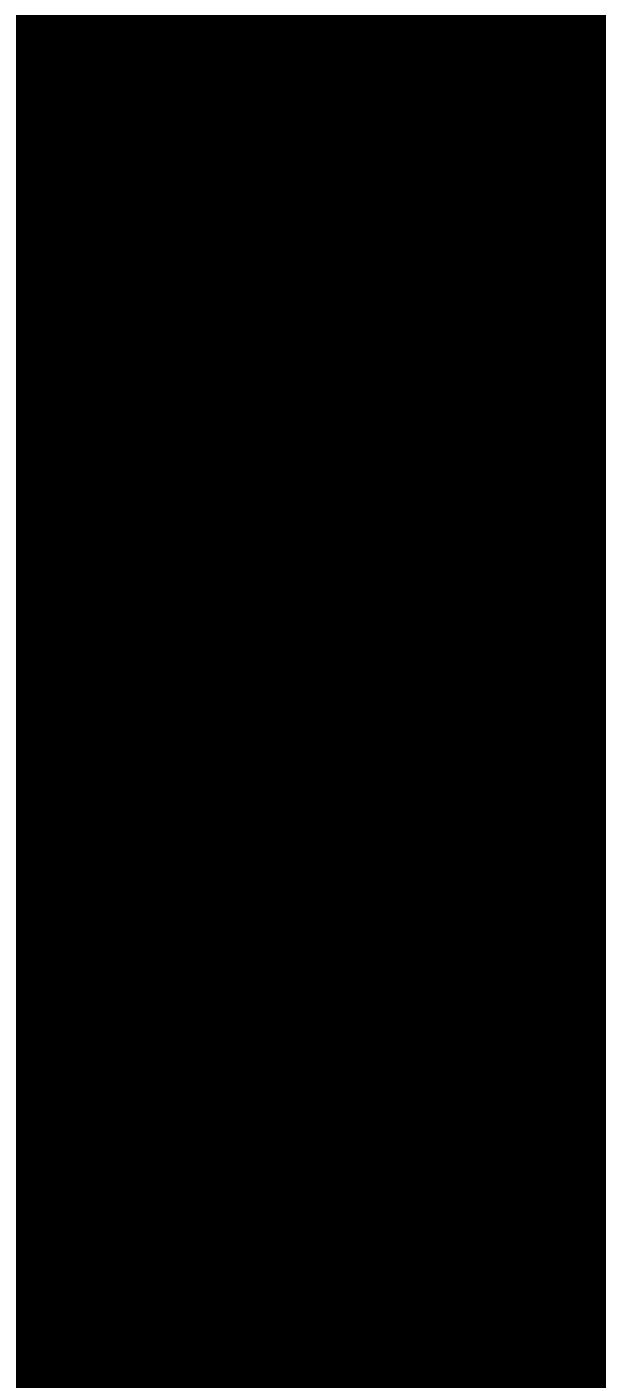

Fig. 7 Typical particle velocity of glass bead as a function of liquid velocity for transport through a foam for various bead diameters: 22.5 $\mu \mathrm{m}$ (circle), $42.5 \mu \mathrm{m}$ (square), and $85 \mu \mathrm{m}$ (diamond). The continuous line of slope is a guide for the eyes and represents $v_{\mathrm{p}}=v_{1}$. 

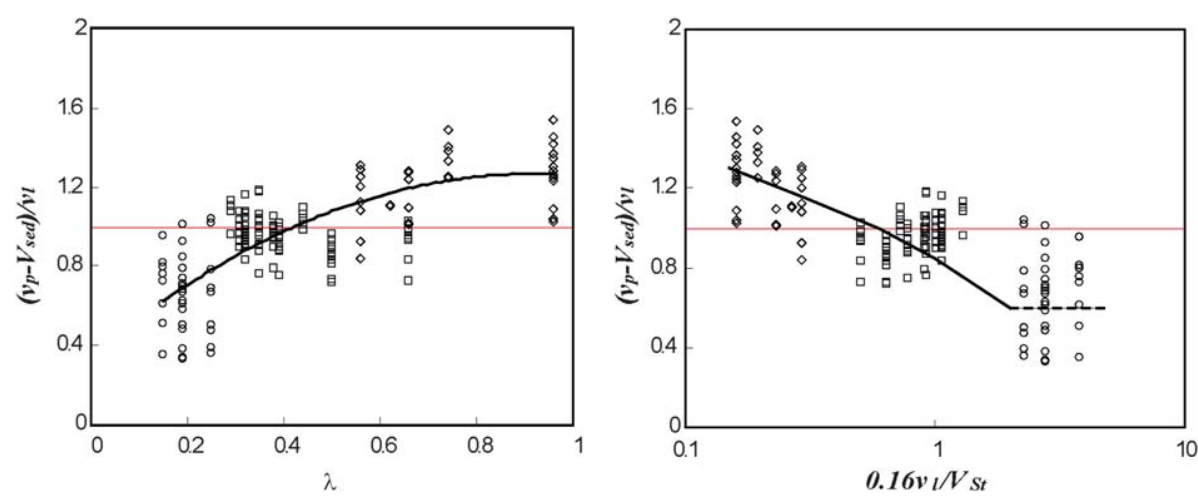

Fig. 8 Normalized particle velocity corrected of sedimentation as a function of confinement parameter $\lambda$ with glass beads in the foam, for three particle diameters: $22.5 \mu \mathrm{m}$ (circle), $42.5 \mu \mathrm{m}$ (square), and $85 \mu \mathrm{m}$ (diamond). The continuous line is a guide for the eyes.

Values for the longitudinal dispersion coefficient are presented in Fig. 9 as a function of $0.16 v_{1} / V_{\mathrm{St}}$. The normalized coefficient of dispersion increases dramatically for $0.16 v_{1} / V_{\mathrm{St}}>1$. We also report data from the literature in Fig. 9. Lee et al. ${ }^{15}$ have shown experimentally that the dispersion of both tracer (colorant) and tiny particles is similar, they find that the ratio $v_{1} R_{\mathrm{PB}} / D_{\mathrm{L}}$ is equal to 0.15 for liquid (tracer colorant) and 0.16 for tiny particles. From their data, we estimate the normalized dispersion coefficient as $\frac{D_{\mathrm{L}}}{v_{\mathrm{l}} 2 R_{\mathrm{b}}}=\frac{D_{\mathrm{L}}}{v_{1} R_{\mathrm{PB}}} \frac{R_{\mathrm{PB}}}{2 R_{\mathrm{b}}}$, respectively, of the order of 1.53 and 1.44 for tracer (colorant) and tiny particles, where $\frac{R_{\mathrm{PB}}}{2 R_{\mathrm{b}}} \approx 0.23$ (from Fig. 7 in ref. 15). These values are reported in Fig. 9 for $0.16 v_{1} / V_{\mathrm{St}} \gg 1$, showing very good agreement with our experimental results for the smallest particles. This agreement is far to be trivial: first, the liquid drainage of Lee's experiments is said to be dominated by viscous dissipation in PB channels which is not the case in the present experiment; second, their scaling of $D_{\mathrm{L}}$ with $R_{\mathrm{PB}}$ is controversial. ${ }^{28,29}$ Note that numerical simulations of Meloy et al. ${ }^{30}$ are consistent with experimental data of Lee et al. ${ }^{15}$ for small particles, but do not predict the strong decrease we observed for the dispersion coefficient of large particles. This disagreement could be attributed to the fact that the authors did not consider the settling of particles in the junctions as a significant effect in this problem.

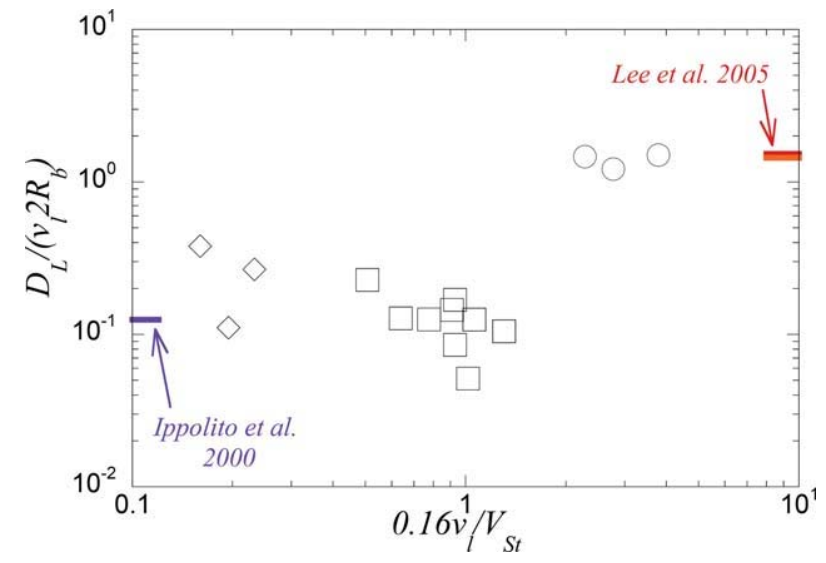

Fig. 9 Normalized dispersion coefficient as a function of the ratio of settling time over convection time in foam junction for three particle diameters: $22.5 \mu \mathrm{m}$ (circle), $42.5 \mu \mathrm{m}$ (square), and $85 \mu \mathrm{m}$ (diamond).
As transport in the foam can be, to a certain extent, compared to transport in solid porous media, it is interesting to report results obtained by Ippolito et al. ${ }^{31}$ when studying experimentally the transport of a single particle through a $3 \mathrm{D}$, dry and random bed of spheres. From their data (Fig. 12 in ref. 31), we estimate a normalized coefficient of dispersion $\frac{D_{\mathrm{L}}}{\langle V\rangle 2 R_{\mathrm{b}}}=\frac{L_{1}}{2 R_{\mathrm{b}}} \approx 0.1$ where $\langle V\rangle$ and $L_{1}$ are respectively the average particle velocity and the dispersion length. We report this value in Fig. 9 for the limit $0.16 v_{1} / V_{\mathrm{St}} \ll 1$. It was shown by Ippolito et al. that the dispersion length (or dispersion coefficient) is independent of the particle size. On the other hand, for foams, a small increase in the dispersion coefficient can be noticed for $0.16 v_{1} / V_{\mathrm{St}}<1$ in Fig. 9 . Note, however, that if we adopt the normalisation for our data with $\left\langle v_{\mathrm{p}}\right\rangle$ instead of $v_{1}$, it reduces the values of the normalized coefficient of dispersion for large particles, and thus minimizes the trend observed in Fig. 9 for $0.16 v_{1} / V_{\mathrm{St}}<1$. Then similarly to the transport of a single particle through a dry random bed of spheres, the dispersion length of buoyant particles in the foam is found to be independent of the particle size for $0.16 v_{1} / V_{\mathrm{St}}<1$.

\section{Conclusions}

New experimental data have been provided for coarse buoyant and non-buoyant particles transported in foam channels.

As a major result, we show that the velocities of small particles (i.e. $1 / 5$ of the maximum particle size) are smaller than the average liquid velocity, whereas large particles are transported at velocities larger than the liquid velocity, independently of sedimentation effects. We show that the Lemlich theory fails to reproduce these results. Better agreement is obtained for small particles using a recent flow model assuming a recirculation (counterflow) liquid velocity at each corner of the foam channels. However, transport velocities of the largest particles are not properly described, indicating that a more refined model is required.

At the scale of the foam, the relevant parameter has been expressed in comparing the particle convection time through a channel junction with the time corresponding to the vertical settling of particles in channels' junctions: $\sim 0.16 v_{1} / V_{\mathrm{St}}$, where $v_{1}$ is the interstitial liquid velocity and $V_{\mathrm{St}}$ is the Stokes velocity. A marked evolution for both the average velocity and dispersion of particles is observed around $0.16 v_{1} / V_{\mathrm{St}} \approx 1$, emphasizing both confinement and buoyancy effects. 


\section{Notations}

\begin{tabular}{|c|c|}
\hline$B o=\mu_{\mathrm{s}} / \mu R_{\mathrm{PB}}$ & $\begin{array}{l}\text { Boussinesq number defined at the scale of the } \\
\text { Plateau border }\end{array}$ \\
\hline$B o^{\prime}=\frac{\mu_{\mathrm{s}}}{\mu d}$ & $\begin{array}{l}\text { Boussinesq number defined at the scale of the } \\
\text { particle }\end{array}$ \\
\hline$d$ & particle diameter \\
\hline$d_{\lim }$ & $\begin{array}{l}\text { maximum diameter of the circle inscribed in } \\
\text { the PB cross-section }\end{array}$ \\
\hline$D_{\mathrm{L}}$ & $\begin{array}{l}\text { coefficient of dispersion in the longitudinal } \\
\text { direction }\end{array}$ \\
\hline$\lambda=d / d_{\lim }$ & confinement parameter \\
\hline & liquid volume fraction \\
\hline$\tilde{K}(\varepsilon)$ & dimensionless foam permeability \\
\hline$R_{\mathrm{b}}$ & bubble radius \\
\hline$R e_{\mathrm{p}}$ & particulate Reynolds number \\
\hline$R_{\mathrm{PB}}$ & Plateau border radius of curvature \\
\hline$v_{\mathrm{p}}$ & velocity of the particle \\
\hline$V_{\mathrm{c}}$ & $\begin{array}{l}\text { dimensionless velocity of the fluid in the corner } \\
\text { in the simulation }\end{array}$ \\
\hline \multirow[t]{3}{*}{$v_{1}$} & $\begin{array}{l}\text { average liquid velocity in the direction of } \\
\text { transport; at the scale of a single Plateau, } \\
\text { experimentally: } v_{1}=(\text { flow rate }) /(\text { cross-sectional } \\
\text { area }) \text {; numerically: }\end{array}$ \\
\hline & $v_{1}=\frac{1}{A_{\mathrm{PB}}} \int_{A_{\mathrm{PB}}} v \times$ if $(v<0) \mathrm{d} A=1$; at the scale \\
\hline & of a foam, $v_{1}=\frac{\tilde{K}(\varepsilon)}{\varepsilon} \frac{\rho g R_{\mathrm{b}}^{2}}{\mu}=(\mathrm{Q} / \mathrm{S}) / \varepsilon$ \\
\hline$V_{1}$ & $\begin{array}{l}\text { dimensionless velocity of the fluid at the inlet } \\
\text { and outlet of a single Plateau border in the } \\
\text { simulation }\end{array}$ \\
\hline$V_{\mathrm{p}}$ & $\begin{array}{l}\text { dimensionless velocity of the particle in the } \\
\text { simulation }\end{array}$ \\
\hline$V_{\mathrm{St}}$ & Stokes velocity \\
\hline$\sigma$ & surface tension \\
\hline$\mu$ & shear viscosity of the solutions \\
\hline$\mu_{\mathrm{s}}$ & surface shear viscosity of the interfaces liquid/air. \\
\hline
\end{tabular}

\section{Acknowledgements}

We would like to thank Elise Lorenceau for fruitful discussions, comments on this manuscript, and encouragement for simulation works. We gratefully acknowledge financial support from Agence Nationale de la Recherche (ANR-05-JCJC-0234-01), E. S.A. (MAP No. A099-108: C14914/02/NL/SH) and the French Space Agency (convention CNES/70980).

\section{References}

1 A. C. Martinez, E. Rio, G. Delon, A. Saint-Jalmes, D. Langevin and B. P. Binks, Soft Matter, 2008, 4, 1531-1535.

2 S. Cohen-Addad, M. Krzan, R. Höhler and B. Herzhaft, Phys. Rev. Lett., 2007, 99, 168001.

3 J. Rubio, M. L. Souza and R. W. Smith, Overview of flotation as a wastewater treatment technique, Miner. Eng., 2002, 15, 139-155.

4 H.-J. Schulze and A. V. Nguyen, Colloidal Science of Flotation, Surfactant Science Series 118, New York, 2004, ch. 1.

5 S. J. Neethling and J. J. Cilliers, Int. J. Miner. Process., 2003, 72, 267.

6 P. Stevenson, S. Ata and G. M. Evans, Chem. Eng. Sci., 2007, 62, 5736-5744.

7 G. D. Miles, L. Shedlovsky and J. Ross, J. Phys. Chem., 1945, 49, 93107

8 A. Saint-Jalmes, Y. Zhang and D. Langevin, Eur. Phys. J. E, 2004, 15, 53.

9 O. Pitois, C. Fritz and M. Vignes-Adler, J. Colloid Interface Sci, 2005, 282, 458

10 E. Lorenceau, N. Louvet, F. Rouyer and O. Pitois, Eur. Phys. J. E, 2009, 28, 293-304.

11 S. A. Koehler, S. Hilgenfeldt, E. R. Weeks and H. A. Stone, Phys. Rev. E: Stat., Nonlinear, Soft Matter Phys., 2002, 66, 040601.

12 R. A. Leonard and R. Lemlich, AIChE J., 1965, 11, 18.

13 V. Carrier, S. Destouesse and A. Colin, Phys. Rev. E: Stat., Nonlinear, Soft Matter Phys., 2002, 65, 061404.

14 O. Pitois, N. Louvet and F. Rouyer, Eur. Phys. J. E, 2009, 30, 27-35.

15 H. T. Lee, S. J. Neethling and J. J. Cilliers, Colloids Surf., A, 2005, 263, 320-332.

16 N. A. Bennani, A. Fujiwara, S. Takagi and Y. Matsumoto, Colloids Surf., $A, 2007$, 309, 7.

17 O. Pitois, C. Fritz, L. Pasol and M. Adler, Phys. Fluids, 2009, 21, 103304.

18 F. Rouyer, C. Fritz and O. Pitois, Soft Matter, 2010, 6, 3863-3869.

19 N. Louvet, R. Höhler and O. Pitois, Phys. Rev. E: Stat., Nonlinear, Soft Matter Phys., 2010, 82, 041405

20 M. E. Staben, A. Z. Zinchenko and R. H. Davis, Phys. Fluids, 2003, 15, 1711-1733.

21 F. Carn, A. Colin, O. Pitois, M. Vignes-Adler and R. Backov, Langmuir, 2009, 14, 7847.

22 R. M. Guillermic, A. Salonen, J. Emile and A. Saint-Jalmes, Soft Matter, 2009, 5, 4975-4982.

23 A. Britan, M. Liverts, G. Ben-Dor, S. A. Koehler and N. Bennani, Colloids Surf., A, 2009, 344, 15-23.

24 J. Goyon, F. Bertrand, O. Pitois and G. Ovarlez, Phys. Rev. Lett., 2010, 104, 128301.

25 S. Guignot, S. Faure, M. Vignes-Adler and O. Pitois, Chem. Eng. Sci., $2010,65,2579$.

26 S. A. Koehler, S. Hilgenfeldt and H. A. Stone, Langmuir, 2000, 16, 6327-6341.

27 D. Weaire and S. Hutzler, The Physics of Foams, Clarendon Press, Oxford, 1999; I. Cantat, S. Cohen-Addad, F. Elias, F. Graner, O. Pitois, F. Rouyer and A. Saint-Jalmes, Les mousses-Structure et Dynamique, Belin, Paris, 2010.

28 F. Rouyer, O. Pitois, E. Lorenceau and N. Louvet, Phys. Fluids, 2010, 22, 043302 .

29 N. Bennani and S. A. Koehler, Soft Matter, 2010, 6, 3841-3850.

30 R. Meloy, S. J. Neethling and J. J. Cilliers, Int. J. Miner. Process., 2007, 84, 185-191.

31 I. Ippolito, L. Samson, S. Bourlès and J. P. Hulin, Eur. Phys. J. E: Soft Matter Biol. Phys., 2000, 3, 227-236. 